\title{
Scope of practice of the breast care nurse: a comparison of health professional perspectives INTRODUCTION
}

BCNs were formally introduced into Australian health services in the late 1990s to facilitate better continuity of care and psychosocial support (Liebert, et al., 2001). Research examining the BCN role (e.g., White \& Wilkes, 1998; Wilkes et al., 1999) as well as the National Breast Cancer Centre (2000) report describes the role of the nurse as working "collaboratively with the treatment team to ensure that women are fully informed, to coordinate care, to offer counselling and support, to facilitate liaison with and referral to other health professionals and to act as a resource for the other members of the treatment team" (p. 91). Research also shows that BCNs are perceived by colleagues in multidisciplinary teams (MDTs) (Amir et al., 2004) and patients (Campbell, et al., 2006; Halkett, et al., 2006) to provide an important service that has a positive impact on quality of care. As well, Amir et al (2004) found a strong positive association between the presence of a breast cancer nurse in the team and overall clinical performance.

Halkett et al. (2006) reported that patients under the care of a BCN emphasised the value of both continuity of support, and availability throughout their experience to discuss treatment options, concerns, to help make decisions, and to prepare for events following diagnosis. Moreover, women who see a $\mathrm{BCN}$ receive more information about treatment, side effects, follow up care and clinical trials (Campbell et al., 2006), get more emotional support, and are more likely to consider having reconstructive surgery (National Breast Cancer Centre, 2000).

While the positive benefits of BCNs in improving patient well-being have been established, there is a consensus in the literature that their role is poorly defined (Amir et al., 2004; Poole, 1996; Yates et al., 2007). Amir et al, for example, found that the role involves considerable interaction and some overlap of roles with other health 
professionals. Jenkins et al. (2001) identified surgeons, oncologists and BCNs as the main providers of different types of information, yet the professional most consistently playing an unseen role was the $\mathrm{BCN}$.

The importance of delineating the boundaries of the $\mathrm{BCN}$ role is highlighted by the National Breast Cancer Centre (2000) report on an evidence based model for Australian practice. BCNs reported they spent $54 \%$ of their time on clinical activities but experienced significant time pressures due to teaching other team members, attending meetings, and completing administrative activities. This raises the question as to what elements of the $\mathrm{BCN}$ role are most important and whether time BCNs spent on different aspects of their role is consistent with how important those aspects of practice are deemed to be. The situation is made more complex as $\mathrm{BCN}$ positions can vary in classification level and working arrangements, with some BCNs engaged full time and others part time, or even one day per week in between other nursing duties (White \& Wilkes, 1998). The variation in positions contributes to the ambiguity surrounding the precise breadth and depth or 'identity' of the professional BCN role. The variation in positions also suggests there may need to be adaptations to the role description to suit particular practice contexts.

Role ambiguity is a major contributor to job-related stress and burnout across a wide variety of occupations, including nursing (for example, Hochwalder, 2007), and to increased workloads (Catt, et al., 2005). Research already demonstrates that health professionals working in cancer care are at risk of burnout (Trufelli et al., 2008). Therefore, to prevent further stress it is important to ensure the roles of professionals working in cancer care are clearly defined.

A related issue is that different health professionals have diverse perceptions of the BCN role. Jenkins et al. (2001) found a discrepancy in perceived role expectations of self and others between different health professionals in MDTs who care for people with 
breast cancer with the greatest discrepancy observed for the specialist breast nurse. Research on other clinical nurse specialist roles has found similar differences. For example, Catt et al., (2005), in a study of multidisciplinary oncology teams, found that the clinical nurse specialist covered the largest number of information areas. In terms of role clarity there was concordance about the information giving role of the different medical specialists but there was less concordance for specialist nurse roles.

With the more widespread use of BCNs, together with rapid changes in the health care environment, it is important to delineate the current scope of practice of the $\mathrm{BCN}$ role and to understand both how BCNs themselves and other health professionals working in cancer care understand the role's scope. The current paper reports the results of two studies which addressed the following research questions:

RQ 1. What is the scope of practice of BCNs and how does this differ across practice contexts?

RQ 2. How do BCNs and other health professionals differ in their perceptions of the role?

In addition, we examined, from both $\mathrm{BCNs}$ and other health professionals' perspectives, the potential challenges to the enactment of the identified $\mathrm{BCN}$ role.

\section{STUDY 1}

Methods

The study was conducted in Queensland, Australia. Nurses were employed in the $\mathrm{BCN}$ role in a variety of settings, including large metropolitan tertiary hospitals, regional hospitals and rural community health centres.

Participants. All BCNs $(\mathrm{N}=18)$ employed by Queensland Health $(\mathrm{QH}$; the state's health department) were initially contacted by email and invited to participate in the study. Two private nurses also volunteered to participate in the study. In addition, contact details 
were provided by $\mathrm{QH}$ for a number of other health professionals (HPs) involved in breast cancer care. The HPs were from both metropolitan and regional staff; all those approached agreed to participate. Through this method a broad volunteer sample of nurses and medical staff was obtained. The final sample included 11 breast care nurses (nine full time; two part time), one surgeon, two nurse unit managers, three nursing directors, and one medical (oncology) director. BCNs were appointed at different levels, generally Level 2 or Level 4 on the 5 point scale used in QH. Level 2 and Level 4 nurses differ in level of qualifications but were normally employed to undertake the same role. The sample was representative of Queensland BCNs regarding level, terms of employment and geographical region.

Procedure. Participants were invited to participate through an introductory email letter. Participants who expressed an interest received further information in a participant information and consent form; those who consented to participate were contacted by telephone.

Semi-structured interviews were conducted by telephone in all cases except one, which was conducted face-to-face. The interviewers used an open ended approach beginning with demographic questions. Then each participant was asked "How would you describe the role of the breast care nurse?" As the interview progressed more specific points were addressed, for example, "What does a typical day in your job (BCN job) involve?" Interviews on average lasted 35 minutes.

Ethics. Ethical approval for this study was given by both the University Ethics Committee and Queensland Health HREC.

Analysis. The digitally recorded interviews were transcribed verbatim. Thematic analysis of transcripts identified themes about the $\mathrm{BCN}$ scope of practice, as well as associated challenges. Themes were initially identified by a member of the research team using the 
complete data set. Each interview was then independently coded and analysed by two different researchers for coding reliability. Differences in coding were resolved through discussion, with agreement reached in all cases. BCN transcripts were then compared to HPs' to identify theme frequency and characteristics. Interviews were also scrutinized for similarities and differences in perceptions of metropolitan and regional BCNs.

\section{Results and discussion}

Emergent themes describing the BCN scope of practice appear in Table 1. The first theme centred on direct patient care, that is, care which could be delivered face-toface or by phone in an inpatient, outpatient or home setting. BCNs were described as the first point of contact for people with breast cancer.

\section{INSERT TABLE 1 ABOUT HERE}

The key elements of direct patient care were providing people with breast cancer with information and education, and providing psychosocial support.

"Providing support, education, information to women with breast cancer and to their families." $\mathrm{BCN} 3$

"An information and support service for women once they're diagnosed with breast cancer; and certainly from that point ... being involved in explaining everything to them and walking through with them as they go through their treatment." BCN5

BCNs also attended, with the person with cancer, consultations with other health professionals.

“...we have like a surgical clinic which is... mostly devoted to breast cancer. So she comes to that and comes in, with a patient's permission, and sits in with them." HP10

The final aspect of direct patient care was wound care.

"I check on the progress of wounds healing, development of complications that sort of thing." BCN7 
Many interviewees mentioned that the role extended beyond the patient stating BCNs also provided information and psychosocial support to patients' families.

"[They] provide wonderful support and advocacy for the client and her support people that helps them, the client and their family, to cope with their diagnosis and to understand what's happening and where to go ... it's the BCN who can help to make that pathway of their diagnosis and treatment easier." HP8

The $\mathrm{BCN}$ role was also described as including a range of other duties. BCNs were responsible for co-ordinating care which included organising referrals to, and appointments with, other health professionals.

"The breast nurse is there to sort of help facilitate that and not to work the problem out in itself but to get in contact with the appropriate people, should they be surgical, oncology, psychiatric, cancer support groups..." HP10

Moreover, the BCN role included direct liaison with other health professionals and advocacy on behalf of the person (although this advocacy aspect of the role was not well defined by interviewees).

"[Her] role is that of patient advocate, in a coordinating fashion in many ways ... and keeping an eye on how the patient is coping and fairing and information they are receiving...being a conduit for the concerns of the patient so that [she] is able to feed back to the appropriate people if there's a problem..." HP10

Other aspects of care co-ordination included administrative duties and attending multidisciplinary meetings. Approximately one third of BCNs and HPs reported that in addition to administrative duties and direct patient care, attendance at MDTs was part of the BCN's typical work day.

"She may be attending meetings in regard to multi-disciplinary meetings to discuss the care of ladies, her clients." HP8

Administration included both paperwork associated with each client, as well as data collection and analysis required by $\mathrm{QH}$. 
"Well if I'm doing something in relation to a patient I have to document it. So if it's appropriate I will get their notes. I've actually created a telephone record here for me in my office.... Then I file it in a folder under the appropriate name." $\mathrm{BCN} 17$

Finally, the BCN role encompassed education of other health professionals and some BCNs saw the role as including co-ordination of support groups for people with breast cancer.

"And the education, there's ward education and that sort of thing as well." HP13

In summary, the majority of participants gave similar descriptions of what the BCN position involved, with a number using the term "the lynchpin". They viewed the position as one of co-ordination of care, education, service provision, provision of psychosocial support, and referral to a number of services for those with breast cancer.

The BCNs' gave broadly similar accounts of their scope of practice, however enactment of the role differed from centre to centre in response to case loads and differing treatment journeys, and in relation to available services at different health centres.

"We have our own chemotherapy team and radiotherapy... so basically we need to create an appropriate co-ordination model for our own patients. We don't need to create pathways for them to move into another facility for treatment." HP16 (metropolitan)

Case load affected perceptions about the performance of the role. The volume of cases in metropolitan areas exceeded those of regional areas, consequently differences in perceptions emerged. BCNs and HPs mentioned that large case loads impacted on the amount of time BCNs were able to spend in one-on-one consultations with individuals and their partners or family. BCNs also commented that higher number of cases in metropolitan centres meant more time spent attending to administrative work, attending to referrals, liaising with HPs and so on. 
"I have good follow-up with my patient because I'm in a smaller area whereas the guys in Brisbane they don't get the same sort of follow up because they have too many patients.” BCN2 (regional)

BCNs working in hospitals in which medical interventions took place also spent time on post-operative visits to the ward. In contrast, enactment of the role for rural BCNs was affected by geography.

"I'd like to be able to see my clients face-to-face more. I find the majority of my time is spent talking to my clients on the phone because of distance.” BCN6 (rural)

BCNs and HPs both viewed the position as one of support to those with breast cancer by providing a contact point for coordination of services, delivering education and for giving psychosocial support. However, there were differences between HPs and BCNs in their perceptions of the breadth of activities involved in the BCN role, with HPs arguing for a narrower role for BCNs.

“... She's really a clinical person and needs to be hands on and in touch with their patients rather than sort of a researcher or a sort of an administrative role. " HP10

"They do a lot of home visits. Is that overusing a resource that should be appropriately delivered elsewhere?... You should have to come in for things and you would never get anybody going to visit you at home for most of those little specialty things... but to be visiting homes and doing things like that, they're also being a community nurse as well." HP12

BCNs and HPs also differed in their perceptions regarding amount of time devoted by BCNs to each aspect of their role. Also, BCNs spoke of their position in more personal terms stating themselves to be a contact for the patient, their family and significant others, or an anchor point for patients to which all elements of care were connected. BCNs emphasised the role involved not only provision of information and medical care, but also psychosocial support the (mostly) women and their partners. As 
well, many BCNs referred to the development of a close relationship with their patients during the facilitation and management of their treatment journey.

"BCNs provide support and information and take a lady throughout their breast care journey speaking at the right level and being a central point of contact from the point of diagnosis beyond...the lady is free to contact them at any point. Be that the day of diagnosis or ten years later."

\section{$\mathrm{BCN} 4$}

"[BCNs provide] care coordination to make sure that things run smoothly for people, that their care is flowing, and that they know where they are going." $\mathrm{BCN} 3$

In contrast, HPs referred to the $\mathrm{BCN}$ position more in organisational or medical terms, stating they were an important part of a multi-disciplinary team, for example. As such, most HPs viewed BCNs as indispensable links between patients and varying medical specialists, community services, and support groups throughout their cancer journey.

"The $\mathrm{BCN}$ is there to get in contact with the appropriate people; be that surgery, oncology, psychiatric, cancer support groups ... being there for the patient. [Our] BCN has a vital role in being involved with the multi-disciplinary team ... as a clinical person and needs to be hands on and in touch with the patient." HP10 BCNs and HPs also differed in their perceptions of the longevity of the patientnurse relationship and the treatment journey, and thus also how long it was necessary for the relationship to be maintained. One notable difference between the views of BCNs and HPs regarding enactment of the role was that most HPs made little reference to the ongoing role of $\mathrm{BCNs}$ post-treatment. By contrast, all $\mathrm{BCNs}$ interviewed stated they were involved in post-treatment care (although such care was not required by all patients). Ongoing contact and care was reported by BCNs to last from between 3 and 18 months and sometimes as long as five years (both telephone and face-to-face meetings). BCNs were more likely to mention ongoing care regarding both physical changes and concerns, 
and psychosocial issues such as concerns about self image and relationship challenges during and after treatment. To help manage such situations most BCNs reported they referred their patients to other services:

"I give them the Queensland Cancer Council booklet on breast cancer, emotions, partners, look good feel better...I spend as long as the patient needs [during the consultation] and then they can call [at any time] if they have any questions." BCN2

The importance of these differences between BCNs' and HPs' perceptions of the $\mathrm{BCN}$ role is highlighted when challenges to fulfilling the role identified by interviewees are considered. Three main challenges were identified: isolation, compassion fatigue and time constraints.

Challenges: Isolation, compassion fatigue and time constraints.

Isolation, both geographical and organisational, was mentioned as a specific challenge. There was concern expressed by a number of HPs regarding the potential role isolation that may result from not being closely associated with a multi-disciplinary team. Interview data showed that at times the role was not clearly associated with any team or department and, as such, some nurses felt detached from a professional network. Even when $\mathrm{BCNs}$ were in regular contact with multiple teams, role autonomy sometimes led to perceived isolation.

"What makes it hard? Probably the solitariness of it.... You know, autonomous position, a one-off in an organisation. And finding a team that you become a part of, where you're actually part of a lot of teams and an important member of each of those teams but not a regular members... that not really belonging anywhere" BCN3

BCNs also frequently mentioned compassion fatigue associated with the traumatic nature of the patient's journey. Many BCNs spoke of a high need for psychosocial support 
largely attributable to the ongoing demands of caring for those with breast cancer (some of whom faced difficult journeys compounded by relationship difficulties).

"I hope I hit the right note...I do say to the [women] right from the beginning I never lie [to you]. If it's bad, I'm going to tell you it's bad, if it's good I'm going to tell you it's good. That can be really hard." BCN4

The nurses' experience of grief was also reported as being an ongoing issue associated with the role, at times necessitating the need for grief counselling. The challenge of developing a relationship with the patient was commonly reported as being potentially difficult, necessitating care and attention to professional and personal boundaries. Moreover, a number of BCNs mentioned difficulty in achieving appropriate, timely closure with the patient-health professional relationship. A particularly difficult situation is deciding what involvement to have when a patient has secondaries.

Compassion fatigue was potentially exacerbated by role and geographic isolation where particularly difficult cases or issues often led to the desire to debrief with a colleague, yet the opportunity to do so was limited. Specifically, BCNs expressed a reluctance to encroach on another health professional's time and hesitance to contact fellow BCNs with distress associated with their role, as it was believed all BCNs worked under similar circumstances and shared similar levels of distress. Where they did access other BCNs it was only through an occasional phone call at times of particular distress or uncertainty. Instead, BCNs often coped alone with their distress despite having access to counselling services as part of their employment with QH. A reason cited for this was that counsellors had a limited understanding of the role and thus much time was spent explaining the role leaving limited time to address specific issue(s).

In addition, BCNs and HPs differed in their perception of who was a BCNs' peer, with implications for managing both the reported isolation and compassion fatigue. Most 
BCNs perceived fellow BCNs as peers, whereas HPs tended to view BCNs as peers. Thus when speaking of needs for professional peer support, BCNs referred to ways in which they do and potentially could seek support from each other whereas most HPs believed BCNs would access QH counselling services or speak with a fellow HP should they have particular concerns. Also, most HPs were less aware of the psychosocial needs of the $\mathrm{BCN}$ position than the BCNs were themselves.

Finally, most BCNs reported time constraints as a major challenge and expressed dissatisfaction with not being able to spend as much time with individual patients as BCNs deemed appropriate. Time constraints were associated with having an increasingly large case load, or limitations associated with a particular position (e.g. part-time) and location (a position that covers a vast geographical area).

I: "What makes your job hard?"

BCN5: "Time. Not enough hours in the day. Certainly in covering the area that I do, the travel certainly impedes in the actually client contact time."

In particular, the administrative aspect of their role presented challenges to time management. As BCNs were not normally provided with administrative support they created and maintained all their patients' clinical records. Such work reduced time spent with patients. A number of BCNs perceived these constraints as detrimental to the performance of their role in general.

\section{STUDY 2}

\section{Method}

Participants. Most QH BCNs were initially contacted at a professional development workshop. BCNs absent from the workshop were invited to participate in the study by email. There was a $100 \%$ response rate from BCNs. In addition, health professionals who participated in Study 1 were recontacted and asked to participate in Study 2. Where HPs were no longer in that position they forwarded the email to the person who replaced them 
or people in a related position. The result was a larger sample of HPs that included both a similar profile of HPs to Study 1 and a cross-section of oncology nurses, who had not participated in Study 1.

The final sample included 26 BCNs (12 full time, 14 part time), and 14 oncology nurses, one nurse unit manager, three nursing directors, one ADON cancer, one manager and one medical oncologist. BCNs were appointed at different levels, generally Level 2 or Level 4 on the 5 point scale used in QH. Eight BCNs worked in metropolitan hospitals, 15 in regional hospitals and three in remote hospitals. HPs were also from metropolitan, regional and remote areas.

Survey. Participants were first asked a number of demographic questions and then responded to survey items developed by the researchers based on themes elicited in Study 1. The items were piloted on a small sample of nurses. Participants rated the importance of 21 elements of practice using a 5 point scale $(1=$ not at all to $5=$ extremely $)$ and then rated the extent to which each of the 21 elements of practice was part of the day to day work of a $\mathrm{BCN}$ using a 7 point scale $(1=$ never to $7=$ always $)$.

Ethics. Ethical approval for this study was given by both the University Ethics Committee and Queensland Health HREC.

Results

The means and standard deviations for BCNs and HPs for the importance of different elements of practice and the extent to which BCNs engaged in different elements of practice are presented in Table 2. Most elements of practice were rated by both BCNs and HPs as very to extremely important (i.e., mean was $>4$ ). The exceptions were two elements of direct patient care (seeing patients at home and attending consultations with patients), administrative elements of the job and organising/attending support groups for patients. 


\section{INSERT TABLE 2 ABOUT HERE}

By contrast, there was more variability in the perception of BCNs and HPs as to the day to day activities of BCNs. Both groups rated elements of direct patient care as usually or always undertaken by BCNs with the exception of seeing patients at home and attending consultations with patients. Both groups also considered that BCNs were less frequently providing support and information to patients' families. The other activity that was reported to be undertaken usually or always was paperwork associated with patients and QH. It should also be noted that the standard deviations for BCNs were higher than those for HPs for many aspects of direct patient care, with the standard deviations suggesting some significant variability in how BCNs are enacting their role.

\section{Discussion}

Consistent with previous research (Amir et al., 2004; Campbell, et al., 2006; Halkett, et al., 2006) both BCNs and HPs regarded the BCN role as vital for the continuity of care. However, our studies suggest the $\mathrm{BCN}$ role continues to be wide-ranging. The elements of practice identified in Study 1 were generally consistent with previous research and focus on co-ordinating care and providing physical and psychosocial support for patients (White \& Wilkes, 1998; Yates, et al., 2007). Moreover, in Study 2 these elements of practice were rated as very important. However, the current study shows that the breadth of the role, at least in the Australian context, goes beyond support for patients and their families and carers, to such activities as educating other health professionals and organising/attending support groups, more in line with the educator and public advocate elements identified by White and Wilkes (1998). What has changed since White and Wilkes' study is that multiple elements of the role have become increasingly normative rather than focus only on psychosocial support of patients. Expansions in the role increase the risk of burnout among a group of clinicians already at risk. 
Study 1 also identified differences between BCNs' and HPs' perceptions of the breadth of the role and the length of time BCNs are involved with patients. HPs in Study 1 viewed the role as having a more limited timeframe and scope, with HPs reporting that the role was primarily clinical and in conjunction with a MDT. Such differences in perceptions of the BCN role may potentially affect MDT functioning, the efficiency and morale of individual team members and, in turn, patients (Jenkins et al., 2001). It is noteworthy that these differences were not as evident in Study 2 where there was substantive agreement as to the most important aspects of the BCN role, as well as the day to day nature of the role. An explanation for this inconsistency is that Study 1 was eliciting from participants the most salient elements of the role to the participant, whereas Study 2 examined participant responses to researcher specified elements of the role. The breadth of the role thus may be less salient to HPs but nonetheless acknowledged as part of the role when presented in a survey. Future research needs to ascertain from the patients' point of view the appropriate length and breadth of care.

The differences in perceptions between HPs and BCNs identified in Study 1 may be particularly important as the elements of practice in which their perceptions differed were elements of practice linked to challenges identified in undertaking the $\mathrm{BCN}$ role. For example, HPs talked less about BCNs providing psychosocial support and thus HPs may be less aware of the potential for compassion fatigue in the job.

The potential breadth of the role and the length of time for which BCNs continue to provide care both have workload implications. HPs mentioned less care post-treatment which may in turn mean they have differing views of the workload of BCNs. It is important that hospitals, and health care systems more generally, provide clear relevant guidelines as to how long care for patients should continue and adequately fund that level of care. Such guidelines will reduce the potential for burnout in BCNs, given 
organisational factors such as adequate staffing, administrative support and doctor-nurse relationships are related to lower levels of nurse burnout (Vahey et al., 2004).

Moreover, there needs to be more acknowledgement of the potentially differing demands of the role depending on geographic location. To date many health services have used the same role description for all BCNs. The results of the current studies are suggestive that role descriptions need to be adjusted to particular contexts. We suggest future research explores in more detail how the role might appropriately vary across different practice contexts. In our study BCNs in rural areas had reduced face-to-face contact with patients and faced the challenge of supporting patients as they negotiate multiple facilities for treatment. In addition, rural $\mathrm{BCNs}$ were more likely to be part-time appointments. The implications of these factors for patient care and satisfaction are not well understood, nor the potential for alternate methods of contact with patients such as on-line support, which is increasingly being used in other areas of health care delivery. In contrast, BCNs in metropolitan areas had both a wider range of responsibilities including post-operative care as well as higher caseloads, with the potential for more limited care being provided to patients.

Both HPs and BCNs were aware of the potential isolation of BCNs and its impact. Again, health care systems need to consider the most appropriate organisational structure. BCNs are meant to be part of a MDT that provide support in a range of ways but this was not always the case in the current studies. However, our findings suggest that teams may need to better understand the scope and boundaries of the $\mathrm{BCN}$ role, as well as their need for adequate administrative and psychosocial support. At the same time BCNs themselves in our study identified the value of receiving support from other BCNs rather than from MDTs or organisation provided counselling. Thus health care systems may 
need to consider a matrix organisational structure for BCNs, whereby they are formally working with both multidisciplinary health care teams and other BCNs.

Strengths and limitations

The strength of the current studies was the use of both in-depth interviews and a survey to enable triangulation of findings. Moreover, the studies used a cross-section of health professionals as well as a diverse sample of BCNs. The limitations were the small sample size in one Australian state and the health professional sample limited to administrators, nurses and doctors. The survey needs to be replicated with a larger sample of BCNs to examine in more detail potential differences according to terms of employment (full-time vs. part-time) and geographic variations in the role, as well as a more diverse sample of those involved in service delivery.

\section{Conclusion}

The scope of practice of Breast Care Nurses is diverse and includes many elements beyond direct patient care. BCNs and HPs differ in their perspectives of the some aspects of the breadth and length of the role with potential implications for patient care and $\mathrm{BCN}$ burnout.

Acknowledgements: This research was supported by a grant from Cancer Australia and $\mathrm{QH}$.

\section{References}

Amir, Z., Scully, J., Borrill, C., 2004. The professional role of breast cancer nurses in multi-disciplinary breast cancer care teams. European Journal of Oncology Nursing $8,306-314$ 
Campbell, D., Khan, A., Rankin, N., Williams, P., Redman, S., 2006. Are Specialist Breast Nurses available to Australian women with breast cancer? Cancer Nursing $29,43-48$.

Catt, S., Fallowfield, L., Jenkins, V., Langridge, C., Cox, A., 2005. The informational roles and psychological health of members in 10 oncology multidisciplinary teams in the UK. British Journal of Cancer 93, 1092-1097.

Halkett, G., Arbon, P., Scutter, S., Borg, M., 2006. The role of the breast care nurse during treatment for early breast cancer: The patient's perspective. Contemporary Nurse 23, 46-57.

Hochwalder, J., 2007. The psychosocial work environment and burnout among Swedish registered and assistant nurses: The main, mediating, and moderating role of empowerment. Nursing \& Health Sciences 9, 205-211.

Jenkins, V. A., Fallowfield, L.J., Poole, K., 2001. Are members of multidisciplinary teams in breast cancer aware of each other's informational roles? Quality in Health Care 10, 70-75.

Liebert, B., Parle, M. D., White, K., Rodgers, A., 2001. Establishing an evidence base for the specialist breast nurse: A model for Australian breast cancer care. Australian Health Review 24, 192-200.

National Breast Cancer Centre, 2000. Specialist Breast Nurses: an evidenced-based model for Australian practice. National Breast Cancer Centre, Camperdown, NSW.

Poole, K., 1996. The evolving role of the clinical nurse specialist within the comprehensive breast cancer centre. Journal of Clinical Nursing 5, 341-349.

Trufelli, D.C., Bensi, C.G., Garcia, J.B., Narahara, J.L., Abrao, M.N., Diniz, R.W., Da Coast, M.V., Soare, H.P., Del Giglio, A., 2008. Burnout in cancer care 
professionals: a systematic review and meta-analysis. European Journal of Cancer Care 17, 524-531.

Vahey, D.C., Aitken, L. H., Sloane, D. M., Clarke, S. P., Vargas, D., 2004. Nurse burnout and patient satisfaction. Medical Care 42, 57-66.

White, K., Wilkes, L., 1998. Describing the role of the breast nurse in Australia. European Journal of Oncology Nursing 2, 89-98.

Wilkes, L., White, K., Beale, B., Cole, R., Tracy, S., 1999. Supportive care for women with breast cancer: Australian nurses' perspective. Nursing and Health Sciences 1, $71-76$.

Yates, P., Evans, A., Moore, A., Heartfield, M., Gibson, T., Luxford, K., 2007. Competency standards and educational requirements for Specialist Breast Nurses in Australia. Collegian 14, 11-30. 